\title{
Lattice Boltzmann method for viscoelastic fluids
}

\author{
Iaroslav Ispolatov ${ }^{1,2}$ and Martin Grant ${ }^{1}$ \\ ${ }^{1}$ Centre for the Physics of Materials, Physics Department, Rutherford Building, McGill University, 3600 rue University, Montréal, \\ Québec, Canada, H3A 278 \\ ${ }^{2}$ Center for Studies in Physics and Biology, Rockefeller University, 1230 York Ave, New York, New York 10021 \\ (Received 24 October 2001; revised manuscript received 15 February 2002; published 3 May 2002)
}

\begin{abstract}
A lattice Boltzmann model for viscoelastic flow simulation is proposed. Elastic effects are taken into account within the framework of a Maxwell model. To test the approach, we estimate the transverse velocity autocorrelation function for a freely evolving system, and find clear manifestations of shear at large frequencies. We then characterize boundary-driven shear waves, and the resonant enhancement of shear oscillations in a periodically driven fluid confined within a capillary. The measured shear-wave dispersion relation is compared to that obtained from the Navier-Stokes equation with a Maxwell viscoelastic term, and good agreement is obtained.
\end{abstract}

DOI: 10.1103/PhysRevE.65.056704

PACS number(s): 02.70.Rr, 61.25.Hq, 47.50.+d, 47.10. $+\mathrm{g}$

Although only slightly more than a decade old, the lattice Boltzmann method [1-4] has already gained the status of a versatile simulation tool for homogeneous and heterogeneous flows in various, often very complex, geometries. In this paper, we generalize the method to include the effects of viscoelasticity, a common property of complex fluids. For example, many polymeric fluids are viscous on long-time scales, but elastic on short-time scales. The characteristic time, or the spectrum of such times, separating these two regimes is determined by intramolecular interactions in the fluid. If the molecules do not have time to "get out of the way" on the time scale of a process, they react rigidly, like a solid. Viscoelasticity on physically relevant time scales is common to fluids made up of complex molecules. Hence, its inclusion makes the range of application of the lattice Boltzmann method wider. A modification of the standard lattice Bhatnagar-Gross-Krook (BGK) model [3,5] has been suggested in Ref. [6], which permits shear wave propagation, an intrinsic feature of viscoelastic fluids. However, it does not include memory of accumulated shear strain, which is a necessary and natural feature of viscoelasticity. To address this, we propose a more general approach, based on a physically transparent Maxwell model of viscoelasticity [7], which exhibits viscoelastic properties and accounts for accumulated stress via an exponentially decaying memory function.

For simplicity, consider a standard six-velocity BGK model on a two-dimensional hexagonal lattice. Generalizations to more sophisticated lattice Boltzmann schemes are straightforward. The evolution equations for an $i$ th channel (corresponding to one of the six directions for velocity) involve the occupation number $f_{i}[3]$

$$
f_{i}\left(\vec{r}+\vec{C}_{i}, t+1\right)=f_{i}(\vec{r}, t)+\lambda\left\{f_{i}(\vec{r}, t)-f_{i}^{e q}(\vec{r}, t)\right\},
$$

where $\lambda$ is a relaxation parameter related to viscosity [1-4], and the equilibrium occupation numbers $f_{i}^{e q}$ are

$$
f_{i}^{e q}=\frac{\rho}{6}\left\{1+2 \vec{C}_{i} \cdot \vec{U}+4\left[\left(\vec{C}_{i} \cdot \vec{U}\right)^{2}-\frac{1}{2} U^{2}\right]\right\},
$$

where $\rho \equiv \sum_{i=1}^{6} f_{i}$, and $\vec{U} \equiv \sum_{i=1}^{6} f_{i} \vec{C}_{i}$ are the equilibrium density and velocity at each lattice site, respectively, and $\vec{C}_{i}$, $i=1 \cdots 6$ are the lattice unit vectors. Performing the Chapman-Enskog expansion, one can show $[3,4]$ that these equations give rise to the Navier-Stokes equation with the correct convective term.

The Maxwell model for viscoelastic media [7] links the elastic part of the stress tensor $\Pi_{i j}^{e l}$ to the rate of strain via a linear equation with exponentially decaying elastic "memory":

$$
\tau \frac{\partial \Pi_{i j}^{e l}}{\partial t}=-\Pi_{i j}^{e l}+\mu\left(\frac{\partial v_{i}}{\partial x_{j}}+\frac{\partial v_{j}}{\partial x_{i}}\right),
$$

where $\mu$ is an elastic coefficient and $\tau$ is a memory time. As the six-velocity BGK model adequately reproduces the Navier-Stokes equation for incompressible fluids (i.e., $\vec{\nabla} \cdot \vec{v}$ $=0$ ), we consider this case. Then the viscoelasticity of the fluid can be taken into account by adding the Maxwell elastic stress as a body force $\vec{F}_{e l}(\vec{r}, t)$,

$$
\vec{F}_{e l}(\vec{r}, t)=\frac{\mu}{\tau} \int_{-\infty}^{t} d t^{\prime} e^{-\left(t-t^{\prime}\right) / \tau} \nabla^{2} \vec{v}\left(\vec{r}, t^{\prime}\right),
$$

within the Navier-Stokes equation. In terms of the ChapmanEnskog expansion $[3,4]$ this elastic term is of the same order as the standard viscous term. Hence, to reproduce the elastic term of Eq. (4) in the corresponding continuous NavierStokes equation, we must add its lattice equivalent to the relaxation term in the lattice Boltzmann equations Eq. (1)

$$
\begin{aligned}
f_{i}\left(\vec{r}+\vec{C}_{i}, t+1\right)= & f_{i}(\vec{r}, t)+\lambda\left\{f_{i}(\vec{r}, t)\right. \\
& \left.-f_{i}^{e q}(\vec{r}, t)\right\}+\frac{1}{3}\left[\vec{F}_{e l}(\vec{r}, t) \cdot \vec{C}_{i}\right] .
\end{aligned}
$$

Here, $\vec{F}_{e l}$ is calculated as in Eq. (4), but with discretized time

$$
\vec{F}_{e l}(\vec{r}, t+1)=\vec{F}_{e l}(\vec{r}, t)[1-1 / \tau]+\Delta \vec{U}(\vec{r}, t) \frac{\mu}{\tau},
$$




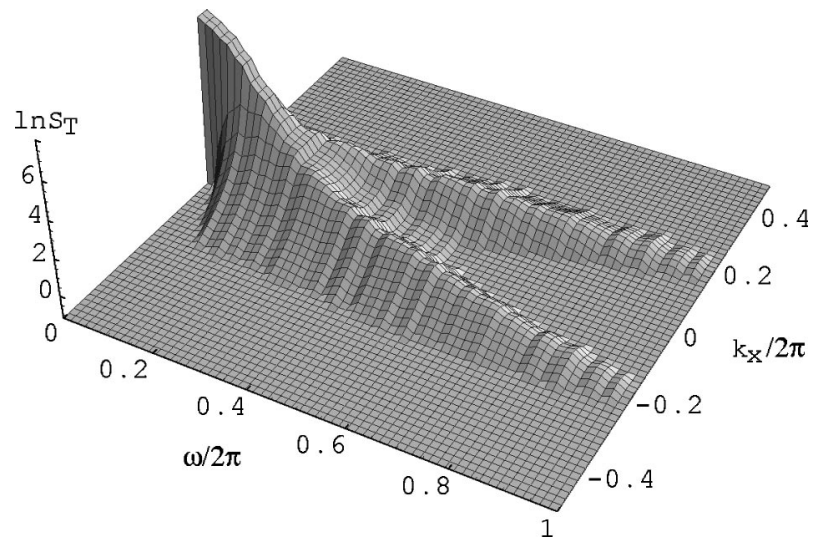

FIG. 1. Sketch of the Fourier transform of the average of the transverse velocity autocorrelation function, $S_{T}\left(k_{x}, \omega\right)$ showing the effect of viscoelasticity. Note the two branches corresponding to shear waves.

where $\Delta$ is the discrete Laplace operator for the hexagonal lattice

$$
\Delta \vec{U}(\vec{r}, t)=\frac{2}{3} \sum_{i=1}^{6}\left[\vec{U}\left(\vec{r}+\vec{C}_{i}, t\right)-\vec{U}(\vec{r}, t)\right]
$$

Equations (5)-(7) formally define our model. Thus, to include the Maxwell viscoelastic effects into the standard lattice Boltzmann method, one need only add a local vector field Eq. (6) to the standard BGK relaxation term Eq. (5), which is updated each time step for every lattice site $\vec{r}$.

To demonstrate viscoelasticity in the model, we first estimate the Fourier transform of the transverse velocity autocorrelation function $S_{T} \equiv\left\langle\left|\widetilde{U}_{y}\left(k_{x}, \omega\right)\right|^{2}\right\rangle$, where the angular brackets denote an average, and

$$
\widetilde{U}_{y}\left(k_{x}, \omega\right) \equiv \frac{1}{L^{2} T} \sum_{x=-L / 2}^{L / 2} \sum_{y=1}^{L} \sum_{t=1}^{T} U_{y}(x, y, t) e^{-i k_{x} x} e^{i \omega t}
$$

Note that $k_{x}=2 \pi n / L, n=-L / 2, \ldots, L / 2$, and $\omega=2 \pi m / T$, where $m=1, \ldots, T$. Here the sum on $y$ corresponds to averaging $\widetilde{U}_{y}\left(k_{x}, \omega\right)$ over the $y$ coordinate. We use the following parameters in our simulation: relaxation parameter $\lambda=$ -1.5 [which corresponds to kinematic viscosity $\nu=1 / 4$ $(-1 / \lambda-1 / 2) \approx 0.042]$, elastic coefficient $\mu=0.3$, memory time $\tau=10$, lattice of $256 \times 256$ sites, maximum time $T$ $=256$, and random initial occupation numbers corresponding to average density per site $\rho=1$. The simulation results, averaged over 100 initial configurations, are presented in Figs. 1 and 2 for the lattice Boltzmann models with and without elastic effects, respectively. Two symmetric branches $\omega$ $=\omega\left(\left|k_{x}\right|\right)$, clearly noticeable in Fig. 1 at large frequencies, correspond to propagating shear waves. These indicate that our model indeed exhibits viscoelasticity.

For more quantitative insight concerning the elasticity, we derive dispersion relations for the continuous shear waves within the framework of the Maxwell model. The linearized

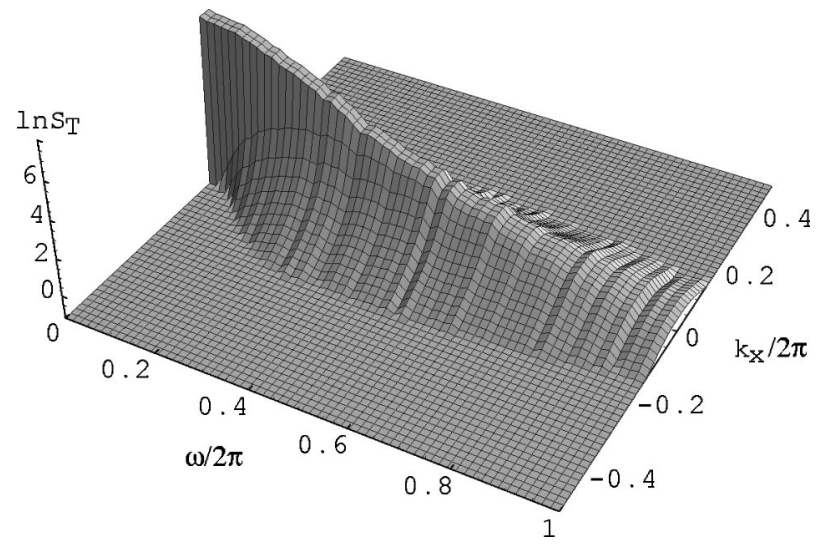

FIG. 2. Sketch of the Fourier transform of the transverse velocity autocorrelation function, $S_{T}\left(k_{x}, \omega\right)$ with no viscoelasticity. Note the absence of shear waves.

Navier-Stokes equation with Maxwell elastic term for transverse velocity of incompressible fluid is

$$
\frac{\partial v}{\partial t}=\nu \nabla^{2} v+\frac{\mu}{\tau} \int_{-\infty}^{t} d t^{\prime} e^{-\left(t-t^{\prime}\right) / \tau} \nabla^{2} \vec{v}\left(\vec{r}, t^{\prime}\right) .
$$

Letting $v(x, t)=v_{0} \exp (-i \omega t) \exp (i k x)$, and discarding exponentially decaying terms, gives

$$
k^{2}=\omega \frac{i+\omega \tau}{\nu(1-i \omega \tau)+\mu} .
$$

On separating the real and imaginary parts of $k, \operatorname{Re}(k)$ and $\operatorname{Im}(k)$, we have

$$
\begin{aligned}
k= & \sqrt{\frac{\omega}{2\left[(\mu+\nu)^{2}+(\nu \omega \tau)^{2}\right]}} \\
& \times\left\{\sqrt{\mu \omega \tau+\sqrt{(\mu \omega \tau)^{2}+\left[\nu\left(1+\omega^{2} \tau^{2}\right)+\mu\right]^{2}}}\right. \\
& \left.+i \sqrt{-\mu \omega \tau+\sqrt{(\mu \omega \tau)^{2}+\left[\nu\left(1+\omega^{2} \tau^{2}\right)+\mu\right]^{2}}}\right\} .
\end{aligned}
$$

We will consider propagating shear waves with small dissipation, so that the ratio

$$
\frac{\operatorname{Re}(k)}{\operatorname{Im}(k)}=\frac{\mu \omega \tau+\sqrt{(\mu \omega \tau)^{2}+\left[\nu\left(1+\omega^{2} \tau^{2}\right)+\mu\right]^{2}}}{\nu\left[1+(\omega \tau)^{2}\right]+\mu}
$$

can be appreciable. Note that $\operatorname{Re}(k) / \operatorname{Im}(k)$ is the dimensionless length of the elastic wave envelope. Naively, one can set $\nu \omega^{2} \tau^{2} \ll \mu$, and choose a sufficiently large value of $\omega \tau$ to make $\operatorname{Re}(k) / \operatorname{Im}(k)$ as large as desired. However, systems with too small a viscosity and too large an elastic coefficient can be numerically unstable. For random initial conditions, or for small drive, we numerically determined that the domain of stability was roughly delimited by $\nu \geqslant 0.04$ and $\mu$ $\leqslant 10 \nu$. For these conditions on viscosity and the elastic coefficient, the largest dimensionless length of the elastic wave envelope is approximately three, which occurs for $\omega \tau \approx 3$. 


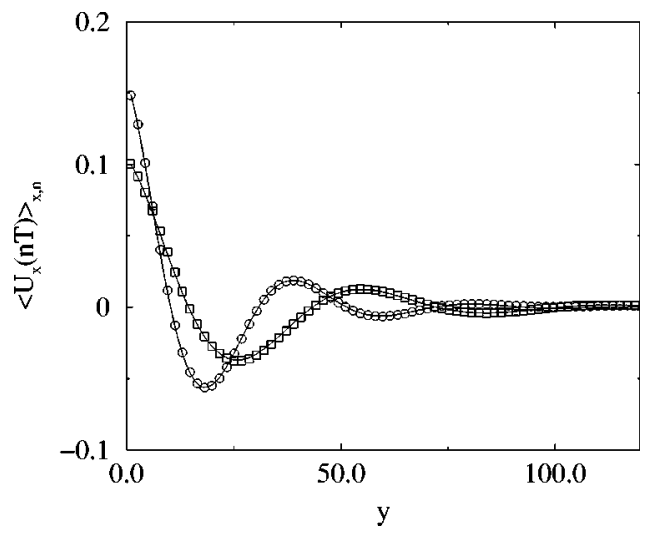

FIG. 3. Plots of the $x$ component of the average velocity $\left\langle U_{x}\left(y, t_{n}\right)\right\rangle_{x, n}$ measured at the times $t_{n}=n T, n=1, \ldots$, vs the distance form the driving wall $y$ for $\nu=0.042$ and $\mu=0.3$. Simulation results for $T=2000, \tau=920$ and $T=4000, \tau=1840$ are shown by circles and squares, respectively. Solid lines correspond to theoretical results in the form $\left\langle U_{x}\right\rangle=A \exp [-\operatorname{Im}(k) y] \cos [\operatorname{Re}(k) y]$.

This gives the dimensionless maximum $\tau$, in terms of frequency, with which the relevance to an experimental system can be determined.

To verify that our model reproduces this, we performed the following simulation. We considered a lattice of size $100 \times 100$, with periodic boundary conditions in the $x$ direction, and reflecting boundary conditions in the $y$ direction. The reflection from the $y=0$ wall is periodically modulated. Therefore, the $x$ components of the velocities after reflection were set proportional to $\cos (\omega t)$. Simulations were performed for $\lambda=-1.5(\nu \approx 0.042)$, elastic coefficient $\mu=0.3$; memory time $\tau=46$ and the period of oscillation $T$ corresponded to the theoretical maximum of $\operatorname{Re}(k) / \operatorname{Im}(k)$.

The simulation results are presented in Fig. 3. This shows the $x$ component of velocity $\left\langle U_{x}\left(y, t_{n}\right)\right\rangle_{x, n}$, measured at the times $t_{n}=n T$, for integer $n$, after averaging $x$ and $n$. Results are shown for $T=2000$ and $\tau=920$ (circles), and for $T$ $=4000$ and $\tau=1840$ (squares). These are compared to the theoretical result $\left\langle U_{x}\right\rangle=A \exp [-\operatorname{Im}(k) y] \cos [\operatorname{Re}(k) y]$, with $\operatorname{Im}(k)$ and $\operatorname{Re}(k)$ as calculated according to Eq. (11). The agreement is excellent for both values of $\tau$.

Finally, consider periodically driven fluid in a capillary. This permits a further quantitative test of our approach. We consider a system of size $9 \times 128$, with stick boundary conditions for the long walls, and periodic boundary conditions for the short walls. A uniform time-periodic volume force $F_{y}=F_{0} \cos (\omega t)$, directed along the longer walls, is applied to the fluid in the capillary. In the update scheme, Eq. (5) is implemented by adding the driving force $F_{y}(t)$ to the elastic force $\vec{F}_{e l}$ in the relaxation term on the right-hand side of the lattice equation.

For a fluid with shear elasticity, there should be a resonance when the driving frequency $\omega$ coincides with the intrinsic oscillation frequency $\Omega$ of elastic media in the capillary. To characterize this resonance, we consider driven oscillations of the first Fourier harmonic of velocity in the direction of the applied force

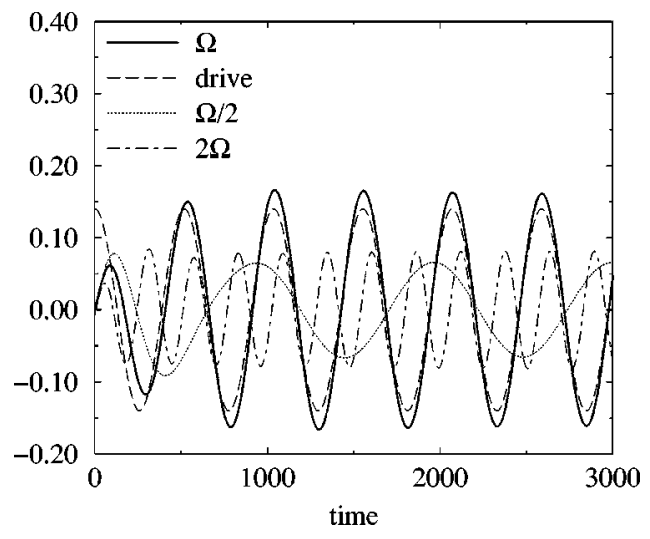

FIG. 4. Amplitude of the first Fourier harmonic $\tilde{v}_{1 y}$ as a function of time for different driving frequencies, $\omega=\Omega, \omega=2 \Omega$, and $\omega=\Omega / 2$. Resonance is clearly apparent for $\omega=\Omega$.

$$
\tilde{v}_{1 y}(t)=\frac{2}{L_{x} L_{y}} \int_{0}^{L_{x}} d x \int_{0}^{L_{y}} d y \sin \left(\pi \frac{x}{L_{x}}\right) v_{y}(x, y, t)
$$

where the integration over $d y$ corresponds to averaging along the length of the capillary. In the presence of a volume force $F_{y}(t)$, a linearized Navier-Stokes equation yields

$$
\tilde{v}_{1 y}=\frac{4 F_{0}}{q L_{x}} \frac{1}{q^{2}\left[\nu+\frac{\mu}{1+\omega^{2} \tau^{2}}\right]+i \omega\left[\frac{q^{2} \mu \tau}{1+\omega^{2} \tau^{2}}-1\right]},
$$

where $q=\pi / L_{x}$ is the wave vector of the first harmonic. Resonance is achieved when the absolute value of this expression has the maximum, i.e., when the second bracket in denominator vanishes

$$
\omega=\Omega \equiv \frac{1}{\tau} \sqrt{q^{2} \mu \tau-1} .
$$

Furthermore, at resonance there should be no phase shift between the driving force and the induced oscillation, so that the ratio between the force and the oscillation amplitudes is real.

To check whether our model behaves as predicted, we performed a simulation using the following parameters: $\mu$ $=0.3, \lambda=-1.5, \tau=250$, and $F_{0}=0.001$. The frequency of the drive $\omega$ was equal to $\Omega \approx 0.011, \Omega / 2$, and $2 \Omega$. The measured values of the $\tilde{v}_{1 y}$ are presented in Fig. 4. After a short transient period the system approaches the steady-state. As anticipated, for resonant drive $(\omega=\Omega)$ the steady state amplitude of oscillation $\tilde{v}_{1 y} \approx 0.16$; while for off-resonance drive, $\omega=\Omega / 2$ and $\omega=2 \Omega$, the amplitudes $\tilde{v}_{1 y}$ are at least twice smaller. To investigate any possible phase shift between the drive and induced oscillations, we also plotted the driving force for the resonant frequency $F(t) \sim \cos (\Omega t)$. One can observe that there is no visible phase shift between driving and induced oscillation. This confirms that the shear elastic resonance frequency of our model is well reproduced by the continuous expression Eq. (15). We also observe that 
when the drive frequency $\omega$ is below or above the resonance frequency $\Omega$, the induced oscillations are phase delayed and phase advanced, respectively, which again follows from Eq. (14).

To conclude, we have proposed a simple yet versatile approach for incorporating viscoelastic effects into lattice Boltzmann simulations for a six-velocity two-dimensional BGK model. Through simulation and analysis for a variety of physical systems, the behavior of the proposed viscoelastic lattice Boltzmann model is qualitatively and quantitatively described by the continuous Navier-Stokes equations with Maxwell viscoelastic term. Hence, we conclude that our model indeed reproduces the Maxwell viscoelasticity for the incompressible fluid flow. Generalization to more sophisticated schemes, including three dimensions and highervelocity models is straightforward.

This work was supported by the Natural Sciences and Engineering Research Council of Canada, and le Fonds pour la Formation de Chercheurs et l'Aide à la Recherche du Québec.
[1] G. R. McNamara and G. Zanetti, Phys. Rev. Lett. 61, 2332 (1988).

[2] F. J. Higuera and J. Jimendes, Europhys. Lett. 9, 663 (1989).

[3] H. D. Chen, S. Y. Chen, and W. Mattheus, Phys. Rev. A 45, R5339 (1992).

[4] S. Chen and G. D. Doolen, Annu. Rev. Fluid Mech. 30, 329 (1998).

[5] The single-relaxation-time collision BGK kernel is due to P. L.
Bhatnagar, E. P. Gross, and M. Krook, Phys. Rev. 94, 511 (1954).

[6] Y. H. Qian and Y. F. Deng, Phys. Rev. Lett. 79, 2742 (1997).

[7] M. J. Crochet, A. R. Davies, and K. Walter, Numerical Simulation of Non-Newtonian Flow (Elsevier Science Publishers, Amsterdam, 1984); R. B. Bird, R. C. Armstrong, and O. Hassager, Dynamics of Polymeric Liquids, Vol. 1, Fluid Mechanics, 2nd ed. (Wiley, New York, 1987). 